\title{
Perfil motor según la batería vítor da fonseca en una población escolar de instituciones educativas del distrito de barranquilla en el año 2018
}

\section{Motor profile according to vítor da fonseca's battery in schoolars of educational institutions of barranquilla district in $\mathbf{2 0 1 8}$}

\author{
DOI: $10.46932 / \mathrm{sfjdv2n2-059}$
}

Received in: january 1st, 2020

Accepted in: March 30th, 2020

\section{Eulalia Amador Rodero}

Fisioterapeuta. Doctora en Metodología de la Investigación Biomédica y Salud Pública. Universidad

Libre - Seccional Barranquilla.

E-mail: eulaliam.amadorr@unilibre.edu.co

\section{Tammy Pulido Iriarte}

Fisioterapeuta. Magíster en Salud Pública. Universidad Libre - Seccional Barranquilla.

E-mail: tammy.pulidoi@unilibre.edu.co

\section{Leslie Montealegre Esmeral}

Fisioterapeuta. Magíster en Salud Pública. Candidata a Doctor en investigación biomédica y salud pública. Universidad Libre - Seccional Barranquilla. E-mail: lesliep.montealegree@ unilibre.edu.co

\section{Emilyn Johana Martínez de la Hoz}

Fisioterapeuta. Egresada Universidad Libre - Seccional Barranquilla

E-mail: emilynj-martinezd@unilibre.edu.co

\section{Sergio Pacheco Carbarcas}

Fisioterapeuta. Egresada Universidad Libre - Seccional Barranquilla.

E-mail: sergioa-pachecoc@unilibre.edu.co

\section{Stefhanie Rivera Modesto}

Fisioterapeuta. Egresada Universidad Libre - Seccional Barranquilla.

E-mail: stefhanie-riveram@unilibre.edu.co

Laura Ardila Pereira

Fisioterapeuta. Mg en Epidemiología. Universidad de Santander.

E-mail: lau.ardila@mail.udes.edu.co

\section{Gina Bustos León}

Fisioterapeuta. Mg en Epidemiología. Universidad de Santander.

E-mail: gin.bustos@mail.udes.co

\section{RESUMEN}

El perfil psicomotor del niño está relacionado directamente con su desarrollo psicomotor, el cual se entiende como un proceso de evolución continua que sucede a medida que van trascurriendo los años. Dicho proceso se da en diferentes etapas, cada una con características específicas, lo cual le permite al niño ir adquiriendo y desarrollando nuevas habilidades 
OBJETIVO: Determinar el Perfil motor según la batería Vítor Da Fonseca en una población escolar de instituciones educativas distritales de Barranquilla. MATERIALES Y MÉTODOS: Se conto con una población de 4867 escolares de las Instituciones Distritales Educativas de la Ciudad de Barranquilla, de la cual se calculo una muestra de 341 inividuos con edades entre 5 a 9 años, escogidos por medio de un muestro aleatorio simple, a los que se les aplico la Batería Psicomotora de Vítor Da Fonseca para evaluar las habilidades y competencias motrices. RESULTADOS: De acuerdo con los resultados obtenidos según la Batería Vítor Da Fonseca se determinó que el $60 \%$ de los niños evaluados tienen su desarrollo motor bueno, el $25 \%$ resultó en una calificación de normal y un $15 \%$ de la población se encuentra dentro de la clasificación de superior. El rango de edad fue de los 5-9 años, con una distribución homogénea, el valor mínimo estuvo para los niños de 5 años conformado por 39 niños $(11,4 \%)$ y la edad promedio fue de 7 años $(24,9 \%)$. Según el sexo 202 de los participantes en el estudio pertenecían al género femenino $(59,2 \%)$ y $139(40,1 \%)$ correspondía al género masculino, por último, según el estrato socioeconómico al estrato socioeconómico, el 38,1\% de los participantes pertenecen al estrato 2 y el 32, $9 \%$ al corresponden al estrato 3 CONCLUSION: Se concluyo que el $60 \%$ de los niños evaluados tienen su desarrollo motor bueno, el $25 \%$ resultó en una calificación de normal y un $15 \%$ de la población se encuentra dentro de la clasificación de superior, dando como explicación que puede que exista una prevalencia del $50 \%$ de la población con alteraciones motoras y un $50 \%$ de no presentar prevalencias en las alteraciones motoras.

Palabras Clave: Desempeño Psicomotor, niño, desarrollo infantil, destreza motora.

\begin{abstract}
INTRODUCTION: The psychomotor profile of the child is directly related to their psychomotor development, which is understood as a process of continuous evolution that happens as the years go by. This process takes place in different stages, each one with specific characteristics, which allows the child to acquire and develop new skills OBJECTIVE: To determine the motor profile according to the Vítor Da Fonseca's battery in schoolars population of Educational Institutions in the district of Barranquilla. MATERIALS AND METHODS A population of 4867 infants was determined, a sample was chosen by means of a random sampling to evaluate the skills and motor skills with the Psychomotor Battery of Vítor Da Fonseca to 341 healthy children with ages between 5 to 9 years of age, of the District Institutions Educational Programs of the City of Barranquilla. RESULTS According to the results obtained according to the Vitor Da Fonseca's battery, it was determined that $60 \%$ of the children evaluated have good motor development, $25 \%$ resulted in a normal rating and $15 \%$ of the population is within the classification of higher. According to the The age range was 5-9 years, with a homogeneous distribution, the minimum value was for children of 5 years consisting of 39 children (11.4\%) and the average age was 7 years (24.9\%). According to sex 202 of the participants in the study belonged to the female gender (59.2\%) and $139(40.1 \%)$ corresponded to the masculine gender, finally according to the socioeconomic stratum to the socioeconomic stratum, $38.1 \%$ of the participants belong to stratum 2 and $32,9 \%$ to correspond to stratum 3. CONCLUSION It was concluded that $60 \%$ of the children evaluated have good motor development, $25 \%$ resulted in a normal rating and $15 \%$ of the population is within the classification of superior, giving as an explanation that there may be a prevalence of $50 \%$ of the population with motor alterations and $50 \%$ of not presenting prevalences in the motor alterations.
\end{abstract}

Keywords: Psychomotor performance, child, child development, motor skills. 


\section{INTRODUCCIÓN}

En los primeros años de vida es importante que los niños se muevan, que interactúen con otros niños/as, que manipulen objetos y que exploren el espacio. Sin embargo, existen altos índices de sedentarismo y falta de horas de psicomotricidad en los centros educativos.

Por lo tanto, esta investigación es de gran importancia para el programa de fisioterapia de Universidad Libre Seccional Barranquilla para promover el aprendizaje de las habilidades motoras de los niños en referencia al Movimiento Corporal Humano. Para nosotros como estudiantes la adquisición de conocimientos acerca de la aplicación de la batería Víctor Da Fonseca y el contacto con una población vulnerable de escasos recursos. La Batería Psicomotora (BPM), es asumida por Vítor Da Fonseca como "un conjunto de situaciones o actividades que procuran analizar dinámicamente el perfil psicomotriz del niño (perfil intra-individual), procurando cubrir su integración psiconeurológica, en concordancia privilegiada con la organización funcional del cerebro propuesta por el psiconeurólogo Luria, para tratar de cuantificar la relación de tal perfil, con su potencial dinámico y su probabilidad de aprendizaje” (1).

El término desarrollo psicomotor (DPM) se atribuye al neuropsiquiatra alemán Carl Wernicke (1848-1905), quien lo utilizó para referirse al fenómeno evolutivo de adquisición continua y progresiva de habilidades a lo largo de la infancia. (Pillmann, Frank. "Carl Wernicke (1848-1905)". (Journal of neurology 250.11 (2003)

Las habilidades mencionadas comprenden la comunicación, el comportamiento y la motricidad del niño. Illingworth (IllingworthR: Dissociation as a guide to developmental assessment. Arch Dis Childhood 1958) aportó una de las definiciones más precisas expresando que el desarrollo psicomotor es un proceso gradual y continuo en el cual es posible identificar etapas o estadios de creciente nivel de complejidad, que se inicia en la concepción y culmina en la madurez, con una secuencia similar en todos los niños, pero con un ritmo variable. (2)

A raíz del desconocimiento del perfil motor de escolares, la investigación será descriptiva, por tanto el problema radica en el conocimiento de ese perfil motor en un grupo de colegios del distrito, como parte del proceso evaluativo que se debería realizar sobre las condiciones de salud específicas para niños en edad escolar de 5-9 años, en donde uno de las problemáticas gira en torno al normal desarrollo motor de niños y niñas en el país, como parte de los direccionamientos de políticas como 0 a siempre, políticas de salud para la infancia, la importancia de identificar tempranamente signos de alarma relacionados con el perfil motor. El propósito de esta investigación es aportar información sobre el perfil motor en los niños de las instituciones educativas distritales de la ciudad de Barranquilla. 


\section{MATERIALES Y MÉTODOS}

\subsection{TIPO DE ESTUDIO}

Descriptivo de corte trasversal, para identificar las características del perfil psicomotor en niños y niñas 5 a 9 años de edad.

\section{POBLACION Y MUESTRA}

La población de este estudio estuvo constituida por 4.687 individuos de 5 a 9 años provenientes de 9 instituciones educativas del Distrito de Barranquilla, de los cuales se calculo una muestra de 341 individuos, escogidos por selección aleatoria simple distribuidos.

\section{INSTRUMENTO}

Las técnica empleada fue la observación directa y el registro. Como instrumento se utilizo la Batería de Vítor Da Fonseca para identificar las características de las habilidades motoras. Da Fonseca es una batería de observación psicomotriz basada en el funcionamiento psicomotor del niño, la edad de aplicación es de 4 a 14 años y evalua en 7 áreas de observación: tonicidad, equilibrio lateralización, noción del cuerpo, estructuración temporo-espacial. Praxia global y praxia fina. La puntuación máxima de la prueba es de 28 puntos (4x7 factores), la mínima es de 7 puntos $(1 \times 7)$ y la media es de 14 puntos. Con base en los respectivos intervalos puntuales puede constituirse una "escala", que apunta a los siguientes valores (ver tabla 1 y 2 ).

Tabla 1. Clasificación Batería Vítor Da Fonseca

\begin{tabular}{|l|l|l|}
\hline \multicolumn{1}{|c|}{$\begin{array}{c}\text { Puntos de la } \\
\text { BPM }\end{array}$} & \multicolumn{1}{|c|}{$\begin{array}{c}\text { Tipo de perfil } \\
\text { psicomotor }\end{array}$} & \multicolumn{1}{c|}{$\begin{array}{c}\text { Dificultades de } \\
\text { aprendizaje }\end{array}$} \\
\hline $27-28$ & Superior & - \\
$22-26$ & Bueno & - \\
$14-21$ & Normal & - \\
$9-13$ & Dispraxico & Ligeras (especificas) \\
$7-8$ & Apraxia & Significativas \\
& & (moderada/severa) \\
\hline
\end{tabular}

Fuente: Vítor Da Fonseca. Manual de observación psicomotriz: significación psiconeurológica de los factores psicomotrices. Primera edición. España: INDE Publicaciones: 1998 
Tabla2. Calificación de las actividades desarrolladas en cada factor, se da de 1 a 4 .

\begin{tabular}{|l|c|c|}
\hline \multicolumn{1}{|c|}{ VARIABLE } & N & $\%$ \\
\hline SEXO & \multicolumn{2}{|c|}{} \\
\hline Femenino & 202 & 59,2 \\
\hline Masculino & 139 & 40,7 \\
\hline EDAD & 39 & 11,4 \\
\hline 5 años & 56 & 16,4 \\
\hline 6 años & 85 & 24,9 \\
\hline 7 años & 82 & 24,0 \\
\hline 8 años & 79 & 23,1 \\
\hline 9 años & \multicolumn{2}{|}{} \\
\hline ESTRATO & \multicolumn{2}{|l|}{} \\
SOCIOECONOMICO & 100 & 29,3 \\
\hline 1 & 130 & 38,1 \\
\hline 2 & 111 & 32,5 \\
\hline 3 &
\end{tabular}

Fuente: Batería Vítor Da Fonseca

\section{RESULTADOS}

Inicialmente se describen los datos sociodemográficos y variables relacionadas con los resultados obtenidos con la batería Vitor Da fonseca con los niños evaluados en las instituciones educativas distritales de la ciudad de Barranquilla.

Tabla $\mathrm{N}^{\circ}$ 3. Distribucion de frecuencia de datos sociodemograficos

\begin{tabular}{|l|c|l|}
\hline \multicolumn{1}{|c|}{ PERFIL } & ANOTACION & \multicolumn{1}{c|}{ COMPORTAMIENTO } \\
\hline APRAXICO O DÉBIL & $\mathbf{1}$ & $\begin{array}{l}\text { Ausencia de respuesta, realización imperfecta, incompleta, } \\
\text { inadecuada y descoordinada débil y muy débil; disfunciones } \\
\text { evidentes y obvias, objetivando dificultades de aprendizaje } \\
\text { significativas) }\end{array}$ \\
\hline $\begin{array}{l}\text { DISPRAXICO } \\
\text { INSATISFACTORIO }\end{array}$ & $\mathbf{O}$ & $\begin{array}{l}\text { Débil realización con dificultad de control y señales desviadas } \\
\text { (débil insatisfactorio; disfunciones ligeras, objetivando } \\
\text { dificultades de aprendizaje). }\end{array}$ \\
\hline $\begin{array}{l}\text { EUPRAXICO O BUENO } \\
\text { HIPERPRAXICO }\end{array}$ & $\mathbf{3}$ & $\begin{array}{l}\text { Realización completa, adecuada y controlada (bueno, } \\
\text { disfunciones indiscernibles, no objetivando dificultades de } \\
\text { aprendizaje). }\end{array}$ \\
\hline EXCELENE & $\mathbf{4}$ & $\begin{array}{l}\text { Realización perfecta, precisa, económica y con facilidades de } \\
\text { control (excelente, óptimo; objetivando facilidades de } \\
\text { aprendizaje) }\end{array}$ \\
\hline
\end{tabular}

Fuente: Elaboración grupo investigador (2018)

Nótese que, en la tabla anterior, que 202 de los participantes en el estudio pertenecían al género femenino $(59,2 \%)$ y $139(40,1 \%)$ correspondía al género masculino. El rango de edad fue de los 5-9 años, con una distribución homogénea, el valor mínimo estuvo para los niños de 5 años conformado por 39 niños $(11,4 \%)$ y la edad promedio fue de 7 años $(24,9 \%)$. Referente al estrato socioeconómico, el 38,1\% de los participantes pertenecen al estrato 2 y el 32, $9 \%$ al corresponden al estrato 3 . 


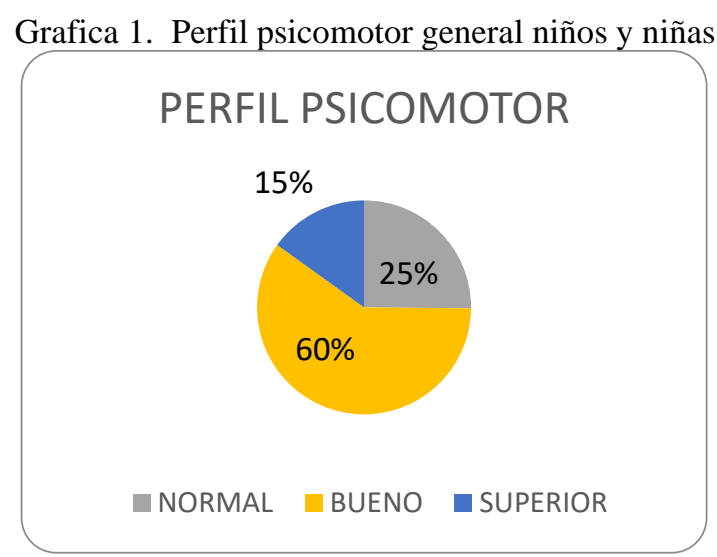

Fuente: Elaboración propia (2018)

Según la gráfica del resultado total de la aplicación de la prueba, se puede extraer que el 60\% de los niños evaluados tienen su desarrollo motor bueno, el $25 \%$ resultó en una calificación de normal y un 15\% de la población se encuentra dentro de la clasificación de superior. De acuerdo con estos resultados encontramos que la evaluación del perfil psicomotor de los niños y niñas de las 9 instituciones educativas distritales de barranquilla se encuentra dentro de los parámetros normales.

\section{DISCUSIÓN}

En algunos países latinoamericanos, se han realizado valoraciones importantes del desarrollo psicomotor, desde los centros de atención. En Argentina, por ejemplo, entre 1988 y 1994, el Servicio de Crecimiento y Desarrollo del Hospital Nacional de Pediatría “Prof. Dr. Juan P. Garrahan”, convocó a más de 20013 pediatras que llevaron a cabo la evaluación del desarrollo psicomotor de 3.573 niños sanos de todo el país (3). En Colombia por su parte, no existen estudios que muestren procesos efectivos frente a la determinación de perfiles psicomotores en niños o escolares, y menos, programas de intervención en niños con bajos perfiles psicomotrices. Sin embargo, sí se han encontrado estudios que dan razón sobre la identificación del perfil psicomotor y la secuencialidad en el desarrollo en las escuelas primarias con aplicación de instrumentos que establecen una categorización y el desarrollo de procesos de intervención multidisciplinario, en el que se destaca el estudio de Vidarte y col (2009), donde a partir de alteraciones como el déficit de atención/hiperactividad (TDAH) afectan la motricidad, determinan el perfil psicomotor de los niños de 5 a 12 años diagnosticados clínicamente con TDAH en la ciudad de Manizales en comparación con niños sanos de la misma edad, este estudio es el que fundamenta la presente investigación con la diferencia particular que será aplicado en niños sanos exclusivamente. (4)

Un estudio realizado en España coincide con los resultados arrojados en esta investigación con respecto al subfactor equilibrio. Aunque en tal caso, se utilizó un instrumento de valoración diferente (Movement $\mathrm{ABC}$ ), se hallaron diferencias significativas en el equilibrio dinámico, que mostraban 
mejores calificaciones en las niñas que en los niños (5). Otro subfactor en el que las niñas superaron a los niños de manera general fue la praxia fina. Esta cualidad biomotriz implica la capacidad del niño para agarrar y manipular objetos con cierta precisión (6). Una de las razones que explicarían un mejor rendimiento en este aspecto en las niñas, es el tipo de juegos que se escogen y se les asignan. En general, los niños son más activos y les gusta explorar y manipular.

Por último, Cabra menciona en su estudio que la batería propuesta por Vítor Da Fonseca permite una adecuada valoración de la motricidad de los infantes en los siete factores relacionados, si se es exigente en el momento de calificar la actividad realizada (3). Sin embargo, se puede considerar que, en el momento de ubicar a los niños y las niñas en el perfil psicomotriz, los rangos establecidos para determinarlo son muy amplios, lo que lleva que la mayoría de los infantes sea ubicada en el rango normal, aun cuando presentan una realización débil o con dificultad de las actividades planteadas; esto se convierte de alguna manera en una limitante en el momento de establecer resultados generales (7). Objetos más que a las niñas; pero las niñas son más sensibles a las condiciones del medio ambiente, de tal forma que realizan juegos menos activos y dedican menor tiempo a estas prácticas en comparación con los niños. De igual forma, los padres son más permisivos con los hijos varones, lo que se traduce en brindarles mayores oportunidades de momentos activos en su quehacer diario (8).

\section{CONCLUSIONES}

Con la realización de este proyecto se pretendía encontrar una relación entre el déficit o alteración de la habilidad psicomotora de los niños de las instituciones distritales de la ciudad de Barranquilla. Se concluyo que el $60 \%$ de los niños evaluados tienen su desarrollo motor bueno, el $25 \%$ resultó en una calificación de normal y un $15 \%$ de la población se encuentra dentro de la clasificación de superior, dando como explicación que puede que exista una prevalencia del $50 \%$ de la población con alteraciones motoras y un $50 \%$ de no presentar prevalencias en las alteraciones motoras. De acuerdo con el El rango de edad fue de los 5-9 años, con una distribución homogénea, el valor mínimo estuvo para los niños de 5 años conformado por 39 niños $(11,4 \%)$ y la edad promedio fue de 7 años $(24,9 \%)$. Según el sexo 202 de los participantes en el estudio pertenecían al género femenino $(59,2 \%)$ y $139(40,1 \%)$ correspondía al género masculino, por último, según el estrato socioeconómico al estrato socioeconómico, el 38,1\% de los participantes pertenecen al estrato 2 y el 32, $9 \%$ al corresponden al estrato 3.

Por otro lado, El movimiento se consideran fundamentales para las experiencias motrices de desarrollo y perfeccionamiento de patrones maduros, el desarrollo de habilidades elementales es un proceso que involucra la maduración y la experiencia. Los padres y profesores pueden desempeñar un papel importante en la identificación de la mejor forma de abordar este tema y cual seria la metodología 
adecuada de intervención, algunos de los puntos a tener en cuenta son: el desarrollo motor durante la niñez temprana debe ser con énfasis en el desarrollo de habilidades fundamentales locomotoras y manipulativas, es importante tener en cuenta que, para la elaboración de un diseño de programa, la observación y evaluación de estos patrones motrices es importante para poder aplicar métodos de enseñanza adecuados (9).

Para nosotros como fisioterapeutas y kinesiólogos este tipo de investigaciones es una oportunidad de crear espacios laborales, académicos en donde estamos en capacidad de desarrollar estrategias adecuadas e integrales con un abordaje diferente al ofrecido en la actualidad, mejorando seguramente la calidad de vida de estos niños y niñas, pertenecientes a población vulnerable, sus familias y seguramente la misma comunidad (9). 


\section{REFERENCIAS BIBLIOGRÁFICAS}

1. Da Fonseca, Vítor. Manual de observación psicomotriz: significación psiconeurológica de los factores psicomotores. Inde, 1998.

2. IllingworthR: Dissociation as a guide to developmental assessment. Arch Dis Childhood 1958; 33 : $118-22$.

3. Cabra CA, Hincapié SM, Jiménez DI, Tobón M. Estudio descriptivo de los efectos que ejerce el perro como mascota en el desarrollo de la motricidad gruesa de infantes sanos de cinco años de edad. Rev. Lasallista Investig 2011; 8 (1): 82-9.

4. Lejarraga H, Krupitzky S, Giménez E, Diament N, Kelmansky D, Tibaldi F. The organization of a national survey for evaluating child psychomotor development in Argentina. Paed Perin Epidemiol.1997;11:359-73

5. $\quad$ Pillmann, Frank. "Carl Wernicke (1848-1905)". Journal of neurology 250.11 (2003): 1390-1391.

6. Pedro Antonio Calero Saa, Hernán Mauricio García Cardona. Perfil Psicomotor De Los Niños En Edades Entre 6 Y 10 Años, pertenecientes aLos Planteles Educativos De La Ciudad De Pereira, 2013.

7. Bustamante A, Caballero L, Enciso N, Salazar I, Teixeira AF, et al. Coordinación motora: Influencia de la edad, sexo, estatus socioeconómico y niveles de adiposidad en niños peruanos. Rev. Bras. Cineantropom. Desempenho Hum. 2008; 10(1): 25-34.

8. Moreno-Gutiérrez Paula Andrea, Ochoa-Orozco Sergio Andrés, Vásquez-Velásquez Diana Salazar-Becerra Diana, Ortiz-Vanegas Erika, López-Pantoja Yuli, Marín-Arango Lina, García-Torres Adriana. Trastornos de la conducta alimentaria en adolescentes de colegios públicos de Pereira. Rev. Méd. Risaralda 2016; 22 (1): 9 - 13.

9. Rodríguez, Angélica Yulieth, G. Becerra, and L. Quintero. Evaluación del factor psicomotor de la estructuración espacio temporal en niños pertenecientes a las escuelas de la ciudad de Pereira, con edades entre 4 a 14 años, basados en la batería de Vítor Da Fonseca. Diss. Universidad Tecnológica de Pereira. Facultad de Ciencias de la Salud. Ciencias del Deporte y la Recreación, 2013.

10. Ruiz LM, Graupera JL, Competencia motriz y género entre escolares españoles. Rev. in. med.cienc. act. fís. deporte. 2003; 3(10): 101-111.Vidarte JA, Ezquerro M, Giráldez MA. Perfil psicomotor de niños de 5 a 12 años diagnosticados clínicamente de trastorno por déficit de atención/hiperactividad en Colombia.Rev. Neurol. 2009; 49 (2): 69-75.

11. Machacon Noguera Luz Mery, García PuelloFlorlinda. Perfil Psicomotor en Niños Escolares: Diferencias de Género. Cienc. innov. salud. Diciembre 2013; 1 (2):108 - 113. Universidad Simón Bolívar (Col). 